\title{
A Chandra study of the morphological structure of the Wolf-Rayet dwarf starburst galaxy NGC 4214
}

\author{
Joanna M. Hartwell, Ian R. Stevens \\ School of Physics and Astronomy, The University of Birmingham, \\ Edgbaston, Birmingham B15 2TT, UK
}

\author{
David K. Strickland, and Timothy M. Heckman \\ Department of Physics and Astronomy, The Johns Hopkins University, \\ 3400 North Carolina Street, Baltimore, MD 21218, USA
}

\begin{abstract}
We present results from a Chandra X-ray observation of the dwarf starburst galaxy NGC 4214, a galaxy containing several young star forming regions. Starburst regions are known to be associated with diffuse X-ray emission, and in this case $\mathrm{X}$-ray emission from the galaxy shows an interesting morphological structure with a bright $\mathrm{X}$-ray ring of emission within the galaxy.
\end{abstract}

\section{Introduction}

NGC 4214 is a starburst galaxy situated at 2.94 Mpc (Maíz-Apellániz, Cieza \& MacKenty 2002) with an almost face-on orientation. The galaxy has undergone several recent starbursts leading to the formation of OB associations and Super Star Clusters (SSCs). HST observations show two large H II complexes as well as filamentary $\mathrm{H} \alpha$ emission (MacKenty et al. 2000).

\section{Diffuse emission}

NGC 4214 was observed using the Chandra-ACIS-S instrument on 22nd October 2001 for $24 \mathrm{ksec}$ (although $\sim 10 \mathrm{ksec}$ were removed during the data cleaning process). The data were analysed using the CIAO software analysis tools, and the spectra were fitted with XSPEC.

The data obtained show the diffuse $\mathrm{X}$-ray emission from the central regions of NGC 4214, and a bright ring of emission with a diameter of approximately $1^{\prime}$ is seen at the centre of the galaxy. This emission is concentrated within the confines of the optical galaxy and may be due to the combination of the shocked winds from young stars within the starburst region. The diffuse Xray emission (excluding the point sources) was fitted with a two temperature absorption spectrum with a soft component of $0.15 \mathrm{keV}$ and a hard component of $3.71 \mathrm{keV}$, and has a luminosity of $8.2 \times 10^{37} \mathrm{erg} \mathrm{s}^{-1}$.

Figure 1 (left panel), shows X-ray contours superimposed onto an $\mathrm{H} \alpha$ image of the galaxy (MacKenty et al. 2000). The centre of the X-ray emission corresponds to the North West (NW) complex of $\mathrm{H} \alpha$ emission observed by MacKenty et al. (2000), however, there is no real correlation between the South East (SE) 

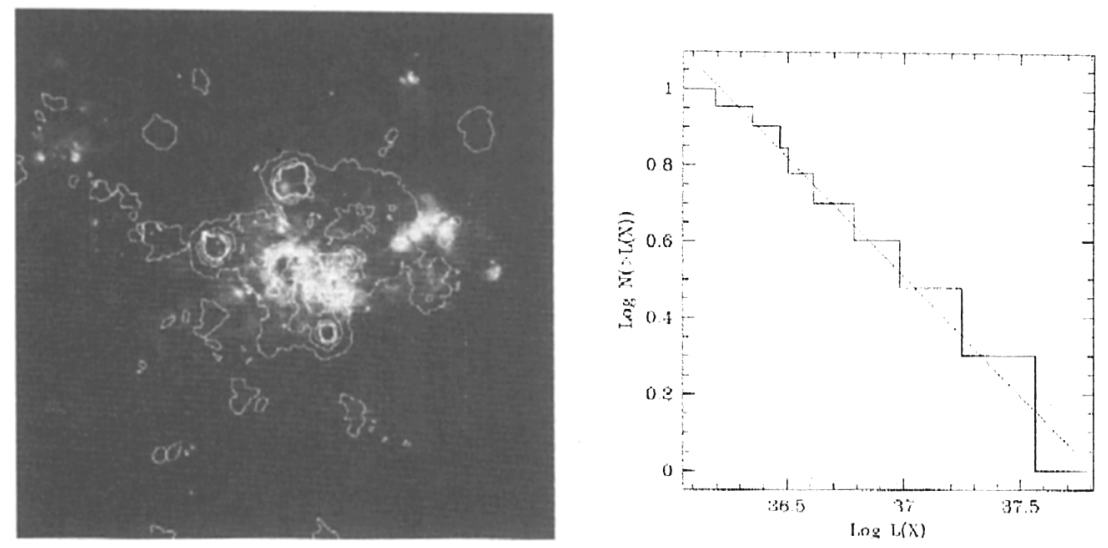

Figure 1. Left: X-ray contours from the binned, smoothed image of NGC 4214 superimposed onto an $\mathrm{H} \alpha$ image. It can be seen that in places the $\mathrm{X}$-ray emission corresponds to regions of increased $\mathrm{H} \alpha$ emission, but that in others there appears to be no correlation. This $\mathrm{H} \alpha$ image was kindly provided by Jesús Maíz-Apellániz from data published in MacKenty et al. (2000) and also available from http://www.stsci.edu/ jmaiz. Right: $\log (\mathrm{N})-\log (\mathrm{S})$ plot for the 11 point sources within the $\mathrm{D}_{25}$ ellipse. The line shown is a power law fit to the data with a slope of -0.61 .

$\mathrm{H} \alpha$ complex and the X-ray emission. This is to be expected due to the fact that the NW complex is older and more evolved than the SE complex, hence it contains more supernovae and the winds of its massive stars have had more time to develop. An X-ray point source is coincident with the dynamical centre of the galaxy, and an extension of X-ray emission corresponds to the position of a known supernova remnant.

\section{Point sources}

Point sources within the $\mathrm{D}_{25}$ ellipse of NGC 4214 were fitted with an absorbed power law model with a photon index of 1.8; from this the flux and hence the luminosity of the sources could be obtained. $A \log (\mathrm{N})-\log (\mathrm{S})$ plot of the data was fitted with a power law with a slope of -0.61 , a value similar to that seen in other starbursts (Figure 1, right panel). However, NGC 4214 covers a lower luminosity range than those previously analysed, and does not show the broken power law fit that is seen in NGC 5253 or NGC 4449 (Summers et al. 2002).

\section{References}

MacKenty, J.W., Maíz-Apellániz, J., Pickens, C.E., Norman, C.A., Walborn, N.R. 2000, AJ 120,3007

Maíz-Apellániz, J., Cieza, L., MacKenty, J.W. 2002, AJ 123, 1307

Summers, L.K., Stevens, I.R., Strickland, D.K., Heckman, T.M. 2002, in preparation 